\author{
Natalia Paprocka (D) \\ Université de Wrocław \\ natalia.paprocka@uwr.edu.pl \\ Justyna Wesola (D) \\ Université de Wrocław \\ natalia.paprocka@uwr.edu.pl
}

\title{
La traduction de l'onomatopée dans les livres pour les tout-petits : la relation texte-image-son
}

\section{Introduction}

\subsection{L'onomatopée, représentation du son}

C'est une chose bien connue : les signes de la langue - matériau sur lequel s'opère la traduction - sont arbitraires. Cela implique qu'il n'existe en principe pas de rapport entre leur forme (signifiant) et leur contenu (signifié). L'auteur de cette observation, Ferdinand de Saussure, reconnaissait toutefois l'existence d'une catégorie de vocables qui permet de la contredire : les onomatopées, représentations des sons inarticulés que sont les bruits de la nature et de la civilisation. Il s'empressa d'ailleurs de préciser que « l'imitation [est] approximative et déjà à demi conventionnelle » et d'en donner un exemple de l'aboiement du chien : « Comparez le français ouaoua et l'allemand wauwau » [Saussure, (1916) $1995: 102]$. 
Il apparaît ainsi qu'un même son peut être perçu, interprété et codifié différemment par les communautés humaines qui parlent des langues différentes. Les onomatopées, résultat de cette codification, deviennent par là-même un objet de traduction extrêmement intéressant, parce que d'une part elles ne diffèrent pas des autres mots (elles sont arbitraires), mais de l'autre, en dépit de leurs formes qui varient selon les langues, elles représentent un même signifié, lequel est - tout comme le langage - un phénomène sonore.

Le terme " onomatopée " signifie « imitation conventionnelle de son réel » [Rittel, $1986: 201]$, mais recouvre plusieurs réalités. Ce peut être : (1) un mot dans lequel on reconnaît l'imitation d'un bruit (par ex.: " fouet », « craquer »), (2) une allitération ou une assonance, répétitions rapprochées de sons identiques visant à produire un effet sonore, ou encore - et est c'est ce qui va nous intéresser ici - (3) une onomatopée proprement dite, " authentique », comme disait Saussure [1995:102], c'est-à-dire un mot invariable, tel boum !, clac ! ou pouf!. En raison de leur forme (mot bref à structure phonologique souvent atypique), on classe souvent les onomatopées proprement dites parmi les interjections, qui leur ressemblent beaucoup (ah!, chut !), même si par nature elles sont tout à fait différentes en termes de sens. Pour dire les choses simplement, le sens d'une onomatopée peut toujours se décrire sous la forme de «bruit de quelque chose » : bruit produit par un être vivant, un mécanisme, un objet, une action ou un phénomène. En revanche, les interjections sont des condensés de message articulé, par exemple : aïe ! signifie « J'ai mal».

\subsection{Les onomatopées dans la littérature}

Comme l'explique Michel Ballard [2001 : 14], les onomatopées se rencontrent surtout dans les comédies, les bandes dessinées et, comme l'ont remarqué aussi Brigitte Schultze et Elżbieta Tabakowska [2004 : 558], dans la littérature enfantine. Lorsqu'elles apparaissent dans des œuvres littéraires plus « sérieuses », c'est généralement dans la bouche d'animaux ou de personnages au bas de la hiérarchie sociale, tels les domestiques ou les enfants [Wesoła, 2008 : 134-135]. Ces derniers emploient spontanément des onomatopées parce que leur vocabulaire est encore relativement pauvre (elles sont donc une sorte de prothèse langagière) [Wesoła, 2008 : 132], et parce que leur attitude face à la réalité est davantage émotionnelle qu'intellectuelle [Schultze, Tabakowska, 2004 : 558]. 
Les onomatopées jouent en général un rôle secondaire dans les textes littéraires, mais il existe une catégorie de livres où elles peuvent se retrouver au premier plan. Ce sont les albums pour les tout-petits (enfants dont l'âge est compris entre 6 mois et 3 ans) ${ }^{1}$, qui ont souvent pour sujet les onomatopées - justement -, en rapport avec le développement du langage chez l'enfant. Celui-ci assimile en effet très tôt ces éléments, entre 1 et 2 ans, au stade mélodique (le premier des quatre stades du développement langagier) [Łobacz, 2005]. L'enfant utilise alors les onomatopées que lui proposent les adultes, mais également crée lui-même des néologismes. En plaçant des onomatopées dans leurs albums pour tout-petits, les auteurs s'adressent ainsi à eux en employant en quelque sorte la langue de leurs lecteurs.

En France, les albums pour enfants en bas âge sont apparus dans les années 1970 et 1980 et représentent aujourd'hui plus de 15\% de l'ensemble des livres pour la jeunesse disponibles sur le marché français [Escarpit, Connan-Pintado, Gaïotti, 2008 : 325-326]. Ils constituent pour les enfants à la fois un « objet éducatif » (puisqu'ils les préparent déjà à la lecture), un " objet ludique » (un jouet) et un « objet affectif» (comme leur ours en peluche). Ils sont destinés à être lus à haute voix par un adulte (qui va lire le livre à et avec son enfant), et cette particularité n'est pas sans importance dans le cas des albums dont la conception repose sur le recours aux onomatopées.

\subsection{La traduction de l'album}

Pour Riitta Oittinen [2008 : 5], l'album (picturebook) est une « forme d'art polyphonique ${ }^{2} »$ dans lequel s'harmonisent plusieurs voix, notamment celle de l'auteur et celle de l'illustrateur. Selon Isabelle Nières-Chevrel, ce concept recouvre deux significations possibles : au sens large, il renvoie à « [...] l'ensemble des livres pour enfants dans lesquels l'image prime sur le texte », et plus étroitement, aux « [...] livres dont les effets de sens reposent sur des interactions du texte, de l'image et du support » [Nières-Chevrel, 2009 : 124]. Dans cette deuxième acception, ce sont des « albums iconotextuels », c'est-à-dire qu'ils sont, avec les bandes dessinées et les romans graphiques, un genre d' « iconotexte ».

\footnotetext{
1 Ces albums sont appelés en anglais early-concept books (voir par ex. Kümmerling-Meibauer, Meibauer, 2011).

2 «[...] a picturebook is a polyhonic form of art ».
} 
Oittinen [2008: 5] caractérise la traduction des albums comme un processus sémiotique au cours duquel le traducteur interprète simultanément le texte et l'illustration. En effet, puisque l'album est un iconotexte, sa traduction se situe à la limite de la traduction interlinguistique et intersémiotique. Son caractère particulier a été souligné également par d'autres traductologues qui se sont intéressés à la question [Ippolito, 2014 : 75 ; Nières-Chevrel, 2003 : 154].

Une seconde source de problèmes dans la traduction des albums vient de l'interdépendance des couches textuelle et sonore. Elle apparaît lorsque l'album est destiné à être lu à haute voix, situation pratiquement inévitable dans le cas des livres pour tout-petits, car ils ne savent pas encore lire euxmêmes. La traduction est alors soumise à un nouveau critère, la fluidité de lecture (fluency), absent dans le cas des livres de lecture silencieuse, ce dont le traducteur est censé tenir compte [Dollerup, 2003 : 100].

\subsection{Objectif et objet de l'étude}

Étant donné leur faible niveau de codification, les onomatopées peuvent représenter un sérieux défi pour les traducteurs. Ce défi gagne encore en importance dans le cas des albums destinés à être lus à haute voix car, comme l'a formulé de manière imagée Riitta Oittinen [2018 : 51], le traducteur est alors appelé à évoluer entre le texte, les images et les sons.

Notre étude a pour objectif d'observer le fonctionnement des onomatopées dans la traduction de ces albums, et ce, sous trois aspects : (1) en relation avec la langue écrite, (2) en relation avec la langue orale, et (3) en relation avec les images qui font partie intégrante de l'album.

Pour cette étude, nous avons analysé Le Livre des bruits (2004), album pour les tout-petits de l'auteure française Soledad Bravi ${ }^{3}$, et ses traductions espagnole (2006) et polonaise (2012). C'est un livre de petit format $(14 \mathrm{~cm} \times 14 \mathrm{~cm})$ mais assez épais en raison de ses grosses pages cartonnées, dont la conception repose sur une cinquantaine d'onomatopées et interjections, à raison de deux pages pour chacun de ces éléments. La page de gauche contient le texte, qui répond chaque fois au même schéma : « X il / elle / ils / elles fait / font Y » (où Y est l'onomatopée / interjection, et X, l'animal, le personnage, la plante, l'objet ou le phénomène

S. Champenois, «Soledad Bravi. Planche de chahut», Libération, le 30 octobre 2012, disponible en ligne : http://next.liberation.fr/livres/2012/10/30/soledad-bravi-planche-de-chahut_857092, consulté le 6 janvier 2018. 
qui produit le son ou qui peut lui être associé). La page de droite contient une illustration de l'élément en question. Mais le livre n'est pas uniquement un recueil d'onomatopées figées, reconnues par l'usage ; il contient aussi des néologismes dans lesquels l'auteure a donné libre cours à son inventivité, et qui, par leur forme atypique, surprennent et amusent le lecteur (le petit comme le grand), surtout lorsqu'ils sont mis en rapport avec l'illustration.

\section{Traduction des onomatopées}

\subsection{Traduction des onomatopées et langue écrite}

Dans son analyse des onomatopées des bandes dessinées de la série Astérix, Xavier Chantry [2001:116] a distingué deux sortes d'onomatopées : les conventionnelles, que l'on retrouve dans les dictionnaires, et les originales, qui sont des néologismes inventés par les auteurs. En adoptant cette distinction, nous avons essayé de définir le caractère des éléments qui apparaissent dans la version originale du Livre des bruits, afin de voir si les traducteurs avaient traduit les premières par des onomatopées traditionnelles, et les secondes par des néologismes de leur cru.

La division des onomatopées en conventionnelles et non conventionnelles appelle une première remarque : le choix des dictionnaires se révèle crucial, car ceux-ci diffèrent par leur choix et leur nombre d'entrées, et peuvent donc prendre en compte ou pas certaines onomatopées. Dans cette situation, il semble judicieux de prendre pour références les dictionnaires canoniques de chacune des langues, car indépendamment de leur culture, ils peuvent être considérés comme équivalents ${ }^{4}$.

D'autre part, se servir des dictionnaires pour déterminer si une onomatopée est conventionnelle ou non est une approche elle aussi conventionnelle. Il semble en effet que ces vocables, en raison de leur caractère marginal, de leur origine primitive et du manque de " sérieux " qu'on leur impute, peinent à entrer dans les dictionnaires classiques. Il est ainsi en réalité difficile de dresser une liste des onomatopées actuelles d'une langue donnée. C'est partiellement compréhensible, car il s'agit d'une catégorie de mots ouverte, qui évolue avec le temps (de ce point de vue,

$4 \quad$ Par ex. trois que nous avons consultés : le Trésor de la langue française, le Stownik języka polskiego de PWN, et le Diccionario de la Lengua Española de l'Académie royale espagnole. La question de savoir à partir de quel moment un dictionnaire est canonique est bien sûr discutable. 
elles se comportent comme les autres parties du langage). Mais les onomatopées sont surtout connues d'un groupe particulier d'usagers, celui des lecteurs de bandes dessinées, et celles qui ne sont pas recensées dans les dictionnaires classiques devraient donc peut-être être considérées comme un vocabulaire de spécialité. C'est ce que semble confirmer le fait que l'on éprouve le besoin de les recenser dans des listes distinctes, telles la «Liste d'onomatopées dans différentes langues ${ }^{5}$ », ou dans des dictionnaires spécialisés (par ex. Gubern, Gasca, 2008 ou Bańko, 2012). Le problème du répertoire d'onomatopées ne semble pas non plus pouvoir se régler entièrement à l'aide des « corpus » linguistiques existants, car ceux-ci ne tiennent pas toujours compte des textes de bandes dessinées, en particulier de ceux qui apparaissent en dehors des bulles.

La « Liste d'onomatopées dans différentes langues » publiée sur Wikipedia n'est sans doute pas aussi prestigieuse ni aussi soignée que les dictionnaires canoniques, mais elle a l'avantage de ne pas s'encombrer de leurs préjugés et de recenser beaucoup plus d'onomatopées. À titre de comparaison, dans l'original du Livre des bruits, on trouve 21 vocables recensés dans le Trésor (en comptant diverses variantes prosodiques et orthographiques d'onomatopées et deux interjections). La « Liste... » en recense 12 de plus (dont une interjection). Deux autres spécimens ne figurent pas dans les dictionnaires, mais peuvent être considérés comme fondés sur des vocables existants (meuh et hmmmm). Bzz bzz et sss ne sont pas recensés dans nos sources, mais correspondent de manière évidente à ce que la conscience collective reconnaît comme le bourdonnement de l'abeille et le sifflement du serpent. Ding dong est un emprunt à l'anglais servant à rendre le son de la cloche, et pouêt est défini dans l'Urban Dictionary en ligne de la manière suivante : " Habituellement utilisé pour évoquer le son des canards en plastique ou d'autres jouets en plastique pour humains ou animaux ou (parfois) les deux $»^{6}$.

Pour résumer, sur les 54 pages de l'album, on retrouve 39 onomatopées ou interjections plus ou moins bien connues des francophones, et

5 « Liste d'onomatopées dans différentes langues », disponible en ligne : https:// fr.wikipedia.org/wiki/Liste_d\%27onomatop\%C3\%A9es_dans_diff\%C3\%A9rentes_ langues, consultée le 6 janvier 2018.

6 «Usually used in the life to speak about the sound of plastic ducks and other plastics toys for humans, pets, or both (sometimes) », Urban Dictionary, disponible en ligne : https://www.urbandictionary.com/define.php?term=Pouet, consulté le 6 janvier 2018 . 
15 créations originales de l'auteure. Certaines sont utilisées à des fins humoristiques et ne peuvent pas être considérées comme propositions de nouvelles onomatopées (par ex. « Noël il fait jingel bells jingel bells » ou « La mouche elle fait tsétsé »).

La comparaison des versions polonaise et espagnole avec l'original montre clairement que les traducteurs n'ont pas restitué systématiquement dans leur langue l'aspect conventionnel ou non des onomatopées. On trouve aussi bien des onomatopées conventionnelles traduites par des formes originales (par exemple, le cri traditionnel de l'âne FR hihan, devient PL $i$-aa $i$-aa, ES $h i$ - $h a h i-h a$, dans deux cas des formes inventées) que l'inverse (le cri de la chouette FR ouh ouh - non conventionnel - devient PL uhuuu - conventionnel ; le bruit de roues du train FR tacata tacata - non conventionnel - devient ES triqui-traca triqui-traca - conventionnel).

On a l'impression que les traducteurs ont soupesé séparément chaque cas d'onomatopée et recherché d'abord un équivalent conventionnel dans leur langue. La comparaison des trois versions montre qu'il existe dans ces trois langues un groupe restreint d'onomatopées de forme fixe et de longue tradition. On y retrouve les bruits d'explosion, l'éternuement, le tic-tac de l'horloge, les bruits de liquides renversés ou qui gouttent, le baiser, et les cris des animaux les plus présents dans l'environnement humain (chien, chat, vache, mouton, chèvre, coq, poule, poussin, coucou). Dans l'original comme dans les traductions, ces onomatopées se présentent sous leur forme du dictionnaire ou légèrement modifiées.

Dans le cas des phénomènes sonores moins fréquents, les traducteurs recherchent des équivalents en se laissant semble-t-il guider à la fois par (1) la forme de l'onomatopée française, (2) les possibilités existantes dans le répertoire lexical de leur langue, et (3) leur image subjective du son à imiter, qui est réalisé ensuite suivant les règles phonétiques et orthographiques propres au polonais ou à l'espagnol.

C'est ainsi par exemple que dans les deux langues romanes concernées, l'onomatopée correspondant au son du tambour recensée dans les dictionnaires est rataplan, que l'auteure et le traducteur espagnol ont tous deux légèrement modifiée : FR ratata plan plan, ES ram-pa-ta-plam. Ils utilisent ainsi le même répertoire de phonèmes (car le $« \mathrm{~m} »$ se prononce [n] dans ce cas en espagnol). Le traducteur polonais a choisi tram tatatam, une forme qui ne rappelle que partiellement l'original puisque la consonne « $\mathrm{p}$ » disparaît, mais qui est proche de la représentation traditionnelle du son du tambour en polonais, présentée par exemple dans 
le Corpus national de la langue polonaise (tram tara ram, ram tam tam). Les analogies de perception des sons dans les langues romanes transparaissent dans d'autres exemples et présentent un contraste marqué par rapport au polonais (voir par exemple le cri de la grenouille : FR coa coa, ES croac croac, PL kum kum).

Les traducteurs essaient généralement d'aligner leurs néologismes sur la forme française (par exemple la voix du fantôme : FR оииииu, PL ииииии, ES иииииh), mais dans certains cas, ils s'en écartent nettement, considérant probablement qu'elle ne sera pas comprise ou acceptée des lecteurs de la traduction. C'est ainsi que le traducteur polonais est plus ou moins d'accord avec l'auteure en ce qui concerne le roucoulement du pigeon : FR rou rou / PL gru gru, alors que l'espagnol se rabat sur une onomatopée proche de celle qui se pratique traditionnellement dans sa langue : cu-curru-cucú.

\subsection{Traduction des onomatopées et langue orale}

Pour comprendre comment fonctionnent dans la réalité les livres destinés à être lus à haute voix, il nous a semblé pertinent d'observer des situations authentiques, où un adulte lit l'album analysé à son enfant. Pour ce faire, nous avons visionné des vidéos publiées sur You Tube qui présentent cette situation précise ${ }^{8}$. Ces films n'ont pas été tournés à des fins métalinguistiques : ce sont de simples souvenirs de famille réalisés par des parents fiers de montrer les progrès de leur enfant.

Avant tout, il semble que le texte comme l'image ne soient, pour l'adulte, qu'un point de départ, et que ce soit lui qui dirige la lecture, en décidant par exemple du temps à passer sur chacune des images. Mais les enfants ne sont pas des auditeurs passifs, bien au contraire : les adultes les

Narodowy Korpus Języka Polskiego, disponible en ligne : http://nkjp.pl, consulté le 6 janvier 2018.

8 Les personnes (adultes et enfants) qui apparaissent dans ces vidéos sont inconnues des auteures du présent article. Pour la facilité, nous appelons les adultes «parents ", même s'il est également possible qu'il s'agisse d'autres proches de l'enfant. Vidéos analysées : «Livre des bruits » : https://www.youtube.com/ watch?v=cGAuUNDqw7k [FR] ; «El libro de los ruidos » : https://www.youtube.com/watch?v=d0vwHm4vj_0 [ES], « Karolina i Księga dźwięków » : https:// www.youtube.com/watch?v=ij5yEiS0q28 [PL1]; " Czytamy księgę dźwięków » : https://www.youtube.com/watch?v=Obqx8G0yadI [PL2]. Dans le texte principal, les chiffres entre crochets indiquent la minute et la seconde du film analysé. 
encouragent à participer. Par exemple le père espagnol - que l'on voit lire tout l'album sur la durée de la vidéo - ne lit en fait que le titre. Ensuite, il ne prononce plus que des substantifs (ou simplement le pronom este [《 lui », " ça »]) et la question ¿cómo hace? (« Il fait comment? ») [par exemple: ES 0:18, 0:34, 0:45].

La lecture de l'album ne se limite pas à une interaction « questions du parent »- « réponses de l'enfant », car les parents commentent aussi les images ou les sons des objets représentés, et bien entendu, ces commentaires ne sont pas écrits dans le livre (par exemple : «Es parecido» [ «C'est pareil »], à propos des sirènes de l'ambulance et de la voiture de police [ES 3:17], ou «El enchufe no se toca » [« La prise de courant, on ne touche pas »] [ES 3:02]). Ces références au monde extérieur laissent entendre que l'album représente des choses qui existent vraiment dans son entourage. Il est donc clair que la lecture de l'album élargit l'horizon de l'enfant et lui fait prendre conscience que le livre est une forme de représentation de la réalité.

L'analyse des vidéos se révèle tout aussi intéressante du point de vue des onomatopées. Sur le site du « Guide de la langue », Mirosław Bańko explique que :

[...] les curiosités linguistiques liées aux onomatopées consistent généralement à dresser des listes de cris d'animaux dans les différentes langues. Sur internet, il est possible de trouver des pages entières de listes de ce genre. On y apprend par exemple que le chien polonais aboie en faisant hau hau, le russe gav gav, le suédois vov vov, et l'anglais, entre autres, bow wow. Sur certains sites, il est même possible d'écouter ces cris d'animaux. Malheureusement, on ne peut pas y écouter la prononciation des mots qui les imitent, c'est-à-dire des onomatopées?.

Dommage que Bańko ne dise pas explicitement pourquoi il le regrette, mais on peut s'en douter. En effet - et nous l'avons déjà signalé -, dans chaque langue, les onomatopées font partie des vocables les moins codifiés. Elles ne sont pas seulement une catégorie de mots ouverte qui accepte facilement les néologismes et dont l'orthographe est fluctuante. Elles sont aussi une image conventionnelle de « sons réels auxquels on

9 M. Bańko, « Onomatopeje », Poradnia językowa, PWN, disponible en ligne : https://sjp.pwn.pl/poradnia/haslo/onomatopeje;7722.html, consulté le 6 janvier 2018. 
associe par convention les phonèmes qui semblent être les plus approchants de ces sons » [Rittel, $1986: 201]$. Ce n'est pas une découverte, mais l'analyse des vidéos fournit des preuves indiscutables en faveur de cette observation.

Dans de nombreux cas, en effet, les onomatopées proposées par l'auteure ou les traducteurs à côté des illustrations ne sont qu'une indication pour le lecteur. Il arrive que celui-ci les lise telles quelles, mais souvent, il opte pour une interprétation personnelle. Le père espagnol imite le son du tambour en disant ram-pam-pam-pam-pam-pam-pam [ES 0:22], alors que dans l'album, il est écrit ram-pa-ta-plam; pour les pétards, il dit pam [ES 0:36] au lieu de bum; pour la mouche, bzt bzt [ES 2:19] au lieu de tsetse. Dans beaucoup de cas, son imagination va bien au-delà de la simple utilisation de phonèmes approchants.

Même dans les cas où il est possible de considérer les phonèmes comme des reproductions exactes, la transcription des onomatopées ne comporte pas d'indications sur leur prosodie. Le chant du coq [ES 3:52], le caquètement des poules [ES 3:58], le cri de l'âne [ES 0:14] et le bêlement du mouton [ES 2:07] sont enrichis dans la vidéo espagnole de traits supplémentaires qui les rapprochent encore davantage du cri réel de ces animaux, et l'imitation par la maman française de la sirène des pompiers [FR 0:11] et de la police [FR 0:20] est criante de vérité.

L'imitation du cochon par le père espagnol [ES 5:02] et le rugissement de lion de la maman française [FR 0:28] sont très clairement des sons inarticulés : il serait impossible de les retranscrire, mais ils ne correspondent certainement pas au oing oing et au rroa rroa imprimés sur les pages de l'album. Ainsi peut-on dire que les lecteurs des onomatopées ne respectent pas strictement la graphie des mots, mais les soumettent en permanence à une analyse critique, en évaluent subjectivement la justesse, et les modifient en fonction de leur propre perception du son.

Signalons encore le commentaire du père espagnol concernant l'onomatopée associée aux épinards. Dans la version française, c'est beurk, une onomatopée employée pour imiter le rot de dégoût à la vue d'une chose peu appétissante. Le sujet hispanophone n'arrive pas à associer à quoi que ce soit le néologisme proposé comme traduction, ecs. Sa réaction est : «Las espinacas no sabemos por qué pero hacen ecs. ¿No? No lo entendemos mucho. » [ES 2:34] («Les épinards, on ne sait pas trop pourquoi, mais ça fait ecs. Non ? C'est pas très clair, tout ça...»). 


\subsection{Traduction des onomatopées et illustrations}

À propos de la spécificité de la traduction des albums, Ippolito a constaté : « Si le traducteur peut produire une série de glissements lexico-sémantiques pour le texte, les images, elles, ne peuvent être soumises à aucune transposition, ni linguistique ni culturelle » [2014: 75]. Certaines ingérences dans les images des albums sont toutefois possibles et se rencontrent parfois, comme le prouve l'analyse des différentes versions linguistiques du livre de Bravi.

Les changements observés dans les images de cet album sont de trois sortes. Les premiers consistent à adapter du texte qui se trouve à l'intérieur d'une illustration, par exemple l'inscription qui apparaît sur la portière d'une voiture de police (POLICE, devenu POLICJA en polonais et laissé tel quel en espagnol) ou sur une boîte de mouchoirs (Mouchoirs - Chusteczki-Pañuelos). Le deuxième type d'ingérence dans l'image consiste à modifier l'illustration même. C'est ainsi que dans la version polonaise, les épinards ne sont plus en boîte mais sur une assiette, et, au bout du canon du pistolet, l'étoile rouge censée représenter le coup de feu est devenue bleue, ce qui peut donner l'impression qu'il s'agit d'eau. L'arme devient ainsi un pistolet à eau, un jouet. Le dernier type d'ingérence dans les images consiste dans un changement d'ordre des illustrations. Encore une fois, c'est dans la version polonaise qu'il se produit : le pistolet et le pétard, qui étaient au tout début de l'album, ont été déplacés au milieu.

Les modifications d'images de types deux et trois ne se retrouvent donc que dans la version polonaise et se rapportent à trois illustrations : les épinards, le pistolet et le pétard. D'autres changements par rapport à la version originale apparaissent encore dans le cas de la main, mais cette fois, se rapportent au texte. Pour en comprendre les raisons, il n'est pas sans importance de signaler que ces quatre illustrations et les textes qui les accompagnent ont suscité d'importantes critiques de la part les lecteurs adultes de la version française, dans laquelle on peut lire : « Les épinards ils font beurk », « Le pistolet il fait pan », « Le pétard il fait boum », et pour l'illustration représentant une main, « La tape elle fait paf ».

Certains lecteurs de l'original français ont estimé que parler d'armes et de violence à de petits enfants ou les inciter à des comportements à risque (jouer avec des pétards) ne correspondait pas à leur vision de l'éducation 
(« Pourquoi apprendre les armes à feu à de très jeunes enfants $?^{10} »$, « Non à la violence éducative présentée comme normale », "C'est à l'inverse du modèle éducatif que j'essaye d'instaurer pour mon enfant »). La vision des épinards présentés d'emblée comme quelque chose de mauvais a également déclenché beaucoup de réactions («Mon fils les aime, pourquoi lui dire que c'est dégoûtant?»).

Les lecteurs espagnols ont eu des réactions semblables. Dans leurs commentaires, on peut lire par exemple : "Certains bruits comme le cachete (« la tape ») ne me paraissent pas convenir aux enfants ${ }^{11} »$. Sur le blog Lactando amando ("Allaiter et aimer »), une lectrice remercie l'une de ses amies de l'avoir mise en garde contre " l'authentique barbarie » (auténtica barbaridad) qu'est à ses yeux le livre de Soledad Bravi. Les lectrices du blog s'insurgent contre les images de la main qui frappe, du pistolet et du biberon. Ce dernier, représenté avec l'onomatopée $m m m m m m$, donne lieu à une critique parce qu'il « ne s'inscrit certainement pas dans le code de déontologie qui promeut l'allaitement naturel $^{12} \gg$. Le son $\mathrm{mmmmmm}$ a donc été interprété comme la manifestation de satisfaction exprimée après avoir goûté à quelque chose de bon, c'est-à-dire comme un message " J'aime bien », et serait par conséquent plutôt une interjection qu'une onomatopée. Toujours dans le même texte, on trouve aussi une critique de deux autres images du livre : celle du bébé qui pleure, et celle du geste du papa pour le faire taire. Mais chose étonnante, les Espagnols ne réagissent pas à la question des épinards. Il se peut que l'onomatopée ecs, qui déroutait déjà le papa de la vidéo, ait été interprétée par erreur comme le bruit que fait la boîte de conserve quand on l'ouvre.

Les Français qui ont lu le livre à leurs enfants ont adopté deux stratégies dans le cas des passages jugés contraires à leurs principes. Certains

10 Tous les commentaires des clients français proviennent du site : https://www. amazon.fr/Livre-Bruits-Soledad-Bravi/product-reviews/2211074227/ref=dpx_ acr_txt?showViewpoints=1, consulté le 6 janvier 2018.

11 « Algunos ruidos como «cachete» no $\mathrm{m}$ [sic!] parecen apropiados para los niños », disponible en ligne : https://www.amazon.es/LIBRO-LOS-RUIDOS\%C3\%81lbumes-ilustrados/dp/8484702324, consulté le 6 janvier 2018.

12 « De hecho seguramente este libro incumpla el código deontológico para la promoción de la lactancia materna », Lactando amando, disponible en ligne : http:// www.lactandoamando.com/2014/08/ellibrodelosruidos.html, consulté le 6 janvier 2018. 
les ont sautés à la lecture (parfois même, en collant les pages pour les « neutraliser » : « Pour la première fois de ma vie, $\mathrm{j}$ 'ai censuré un livre en collant 3 pages entre elles »), d'autres ont remplacé les onomatopées par des formes inoffensives (par exemple, pour les épinards : « miam miam », «mmmhh» ou « humm », et pour la main : « guili » ou « coucou ») ou ont modifié le texte d'une autre façon (par exemple : "Les épinards en boîte font beurk », par opposition aux épinards frais, qui font « miam »).

L'éditeur polonais a réagi dans le même sens que les lecteurs français et espagnols. Le pistolet et le pétard ont donc été déplacés à un endroit moins exposé, et le premier a en outre cessé d'être une arme pour devenir un jouet. Le dessin de la main, qui a déclenché de vives réactions chez les lecteurs français et espagnols, a été « neutralisé » dans la version polonaise grâce à une modification du texte : "Ręce robia klap klap » («Les mains font clap clap »). Alors que tape en français et cachete en espagnol sont compris comme un " coup donné avec la main » et impliquent une idée de violence, les mains mises au pluriel en polonais (ręce) évoquent avant tout un applaudissement, un jeu. Chose curieuse en revanche, si l'éditeur polonais a bien apporté une modification à l'image des épinards (il a remplacé les épinards en boîte, inconnus des enfants polonais, par la panade verte qu'ils sont habitués à voir dans leur assiette), il n'a rien fait pour « corriger » l'interjection de dégoût : elle a été traduite par ble, dont le sens est identique à celui du beurk français.

Ce choix peu pédagogique n'a pas échappé aux lecteurs polonais, qui ont émis des commentaires de la même veine que les parents français ( « Elle n'est pas très 'santé', la page avec les épinards, pas vrai ? $^{13} »$ ). Quant au pistolet et au pétard, les manipulations de l'éditeur n'ont pas empêché les parents polonais de les remarquer malgré tout («Je suis pacifiste et les pistolets n'ont rien à faire chez moi. Pareil pour le pétard, j'espère que Wronek [surnom de l'enfant] ne s'y intéressera pas de sitôt $\left.t^{14} »\right)$, et même d'annoncer leur intention de les censurer (« Je vais en coller quelques-unes, tout simplement. À la colle. Et tant que ce n'est pas fait, je

13 « Troszkę mało prozdrowotna ta strona ze szpinakiem, prawda? », http://wronek. pl/najlepsze-i-najgorsze-strony-ksiegi-dzwiekow/, consulté le 6 janvier 2018.

14 « Jestem pacyfistką i żadne pistolety u nas w domu nie mają racji bytu. Podobnie - lepiej, żeby Wronek [przezwisko dziecka] jak najdłużej nie interesował się petardami », ibidem. 
m'arrange pour sauter les pages $\left.{ }^{15} »\right)$. En revanche, pour la main, le choix du pluriel qui transforme la claque en applaudissement dans la version polonaise est une opération parfaitement réussie : aucun lecteur n'a émis d'objections concernant cette page.

Pour terminer, signalons que l'éditeur français a tenu compte des remarques critiques des lecteurs. Dans la version poche du Livre des bruits parue en 2016, les pages à problème ont été supprimées.

\section{Conclusion}

Dans cette étude, nous avons observé le fonctionnement des onomatopées dans un album pour enfants de moins de trois ans destiné à être lu à haute voix et dont la conception repose sur les onomatopées. Nos observations nous ont permis de distinguer trois aspects des onomatopées qui posent des problèmes de traduction : la nécessité de leur donner une forme écrite dans la langue cible, la grande indépendance de leur prononciation par rapport à la forme écrite, et la grande interdépendance de l'illustration et de l'onomatopée en termes de sens.

Pour traduire les onomatopées, les traducteurs ont tenu compte des équivalents offerts par la langue cible : quand il y en avait, ils les ont adoptés, et dans le cas contraire, ils ont créé de nouvelles formes en se basant sur leur propre perception du son-signifié et en se laissant guider par la graphie de l'original. Lorsqu'ils jugeaient cette dernière acceptable, ils l'ont adaptée à l'orthographe de leur langue, tout en conservant une certaine liberté de répétition de lettres ou de syllabes à l'intérieur de l'onomatopée.

Notre analyse confirme également que les onomatopées de la langue écrite sont purement conventionnelles, non seulement en regard des sons réels qu'elles représentent, mais aussi par rapport à la façon dont on les prononce. Les adultes qui les lisent à leurs enfants les analysent à leur manière. Ce sont eux qui orientent la lecture de l'album et déclenchent les réactions de l'enfant en lui donnant conscience du rapport existant entre l'album et le monde réel. Il leur est loisible de prononcer les onomatopées telles que leur graphie le suggère, de produire un son inarticulé, ou encore d'enrichir leur prononciation de traits prosodiques que le système d'écriture traditionnel est incapable d'indiquer. Les onomatopées « lues »

15 «[...] kilka z nich zamierzam po prostu skleić. Klejem. Dopóki mój zamiar nie zostanie zrealizowany, po prostu staram się je omijać », ibidem. 
à l'enfant sont donc souvent des imitations directes de sons, et elles sont suggérées autant par le texte que par l'illustration.

Les illustrations qui accompagnent les onomatopées ont parfois subi des modifications. En effet, outre les traductions d'inscriptions qui apparaissent dans les images mêmes, on remarque que certaines illustrations dans la traduction polonaise ont été modifiées, probablement à la suite de réactions des lecteurs de la version originale qui ont tenu lieu de censure ou de « contrôle pédagogique ». Ces changements ne se sont cependant révélés que partiellement efficaces, car certains des passages critiqués en français ont aussi entraîné des commentaires des lecteurs polonais.

Cette dernière observation peut surprendre. En effet, dans certains cas, il semble que les lecteurs adultes aient perçu les onomatopées non pas comme de simples bruits de choses ou de personnes, mais comme des représentations de stéréotypes négatifs qu'ils souhaitent éviter dans l'éducation de leurs enfants. Les problèmes perçus dans l'album analysé se rapportaient à la violence, à l'alimentation et aux modèles d'éducation. Ils révèlent à quel point un petit livre pour enfants peut être porteur d'idéologie ou d'une vision du monde. Dans la lignée du titre d'un article de Oittinen [2006] qui signale que la traduction pour enfants n'est pas un acte innocent, nous pouvons constater que le choix et la traduction des onomatopées, formes apparemment anodines, ne le sont pas non plus.

\section{Bibliographie}

\section{Albums analysés :}

Bravi, S. (2004), Le livre des bruits, L'école des loisirs, Paris.

Bravi, S. (2006), El libro de los ruidos, trad. R. Ros Sierra, Editorial Corimbo, Barcelona.

Bravi, S. (2012), Księga dźwięków, trad. M. Byliniak, Dwie Siostry, Warszawa.

\section{Vidéos analysées :}

FR : « Livre des bruits », https://www.youtube.com/watch?v=cGAuUNDqw7k.

ES : «El libro de los ruidos », https://www.youtube.com/watch?v=d0vwHm4vj_0.

PL1 : «Karolina i Księga dźwięków », https://www.youtube.com/watch?v= ij5yEiS0q28.

PL2 : " Czytamy księgę dźwięków », https://www.youtube.com/watch? $\mathrm{v}=$ Obqx8G0yadI. 


\section{Littérature critique :}

Ballard, M. (2001), « Onomatopée et traduction », dans : Ballard, M. (dir.), Oralité et traduction, Artois Presses Université, Arras, pp. 13-42.

Chantry, X. (2001), "Traduction polonaise de l'univers sonore d'Astérix », dans : Skibińska, E. (dir.), Traduction pour la jeunesse face à l'Altérité, Dolnośląskie Wydawnictwo Edukacyjne, Wrocław, pp. 113-127.

Dollerup, C. (2003), « Translation for Reading Aloud », Méta, 48(2), pp. 81-103, https://doi.org/10.7202/006959ar.

Escarpit, D., Connan-Pintado, Ch., Gaïotti, F. (2008), « L'album au tournant du $\mathrm{XXI}^{\mathrm{e}}$ siècle: sous le signe de l'hybridité », dans : Escarpit, D. (dir.), La littérature de jeunesse. Itinéraires d'hier à aujourd'hui, Magnard, Paris, pp. 302-331.

Ippolito, M. (2014), « Traduire les albums pour enfants : Relation texte-image dans les traductions italienne et française de In the Night Kitchen », Traduire. Revue française de la traduction, 230, pp. 75-86, https://doi.org/10.4000/ traduire.627.

Kümmerling-Meibauer, B., Meibauer, J. (2011), « Early-concept Books. Acquiring Nominal and Verbal Concepts », dans : Kümmerling-Meibauer, B. (dir.), Emergent Literacy. Children's Books from 0 to 3, John Benjamins, Amsterdam, Philadelphia, pp. 91-114, https://doi.org/10.1075/swll.13.08kum.

Łobacz, P. (2005), « Prawidłowy rozwój mowy dziecka », dans : Gałkowski T., Szeląg E., Jastrzębowska G. (red.), Podstawy neurologopedii, Wydawnictwo Uniwersytetu Opolskiego, Opole, pp. 231-268.

Nières-Chevrel, I. (2003), «Traduire In the Night Kitchen, ou de la difficile lecture d'un album », Méta, 41(2-3), pp. 154-164, https://doi.org/10.7202/006964ar.

Nières-Chevrel, I. (2009), Introduction à la littérature de jeunesse, Didier Jeunesse, Paris.

Oittinen, R. (2006), « No Innocent Act: On the Ethics of Translating for Children », dans : Van Coillie, J., Verschueren W.P. (eds), Children's Literature in Translation: Challenges and Strategies, St. Jerome, Manchester-Kinderhook, pp. 35-46.

Oittinen, R. (2008), « Audiences and Influences: Multisensory Translations of Picturebooks », dans : González Davies, M., Oittinen, R. (eds), Whose Story? Translating the Verbal and the Visual in Literature for Young Readers, Cambridge Scholars, Newcastle upon Tyne, pp. 3-18.

Oittinen, R. (2018), " The Translator between Images, Words and Sounds », dans : Oittinen, R., Ketola, A., Garavini M. (eds), Translating Picturebooks: 
Revoicing the Verbal, the Visual and the Aural for a Child Audience, Routledge, pp. 51-95, disponible en ligne : https://doi.org/10.4324/9781315112480-3.

Rittel, T. (1986), « Wyrażenia onomatopeiczne w języku polskim (próba opisu morfosyntaktycznego) », Rocznik Naukowo-Dydaktyczny. Wyższa Szkoła Pedagogiczna w Krakowie. Prace Językoznawcze, pp. 201-216.

Saussure, F. de (1995), Cours de linguistique générale, Payot \& Rivages, Paris

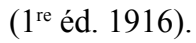

Schultze, B., Tabakowska, E. (2004), «Interjections as a translation problem », dans : Übersetzung - Translation - Traduction, t. 1, Berlin, pp. 555-562.

Wesoła, J. (2008), « Onomatopeyas convencionales españolas en traducciones polacas de obras dramáticas », Romanica Wratislaviensia, 55, pp. 131-144.

\section{Dictionnaires :}

Bańko, M. (2012), Słownik onomatopei czyli wyrazów dźwięko- i ruchonaśladowczych, PWN, Warszawa.

Gubern, R., Gasca, L. (2008), Diccionario de onomatopeyas del cómic, Cátedra, Madrid.

Trésor de la langue française, disponible en ligne : http://atilf.atilf.fr/.

Słownik języka polskiego de PWN, disponible en ligne : https://sjp.pwn.pl/.

Diccionario de la Lengua Española de l'Académie royale espagnole, disponible en ligne : https://dle.rae.es/.

Urban Dictionary, disponible en ligne : https://www.urbandictionary.com/define. php?term=Pouet.

\section{Sites :}

Bańko, M., « Onomatopeje », Poradnia językowa, PWN, disponible en ligne : https://sjp.pwn.pl/poradnia/haslo/onomatopeje; 7722.html.

Champenois, S., «Soledad Bravi. Planche de chahut », Libération, le 30 octobre 2012, disponible en ligne: http://next.liberation.fr/livres/2012/10/30/ soledad-bravi-planche-de-chahut_857092.

«El libro de los ruidos (Álbumes ilustrados): Amazon.es : Soledad Bravi : Libros ", disponible en ligne : https://www.amazon.es/LIBRO-LOSRUIDOS-\%C3\%81lbumes-ilustrados/dp/8484702324.

« Lactando amando. Crianza feliz : El "libro de los ruidos" », disponible en ligne : http://www.lactandoamando.com/2014/08/ellibrodelosruidos.html.

" Liste d'onomatopées dans différentes langues », disponible en ligne : https://fr. wikipedia.org/wiki/Liste_d\%27onomatop\%C3\%A9es_dans_diff $\% \mathrm{C} 3 \%$ A9rentes_langues. 
«Le Livre des Bruits. Commentaires client », disponible en ligne : https:// www.amazon.fr/Livre-Bruits-Soledad-Bravi/product-reviews/2211074227/ ref $=$ dpx_acr_txt?showViewpoints $=1$.

« Najlepsze i najgorsze strony "Księgi Dźwięków" - Wronek », disponible en ligne : http://wronek.pl/najlepsze-i-najgorsze-strony-ksiegi-dzwiekow/. Narodowy Korpus Języka Polskiego, disponible en ligne : http://nkjp.pl.

\section{RÉSUMÉ}

L'objectif de l'étude est d'observer le fonctionnement des onomatopées dans la traduction des albums pour enfants en bas âge (moins de trois ans). Les couches textuelle, visuelle et sonore demeurent, dans ces livres, dans une grande interdépendance et leur conception repose souvent sur les onomatopées, ce qui est en rapport avec le développement du langage chez l'enfant.

En prenant comme exemple Le Livre des bruits de Soledad Bravi et ses traductions espagnole et polonaise, nous avons distingué plusieurs problèmes de traduction liés (1) à la nécessité de donner aux onomatopées une forme écrite dans la langue cible, (2) à l'indépendance de leur prononciation par rapport à la forme écrite, et (3) à l'interdépendance de l'illustration et de l'onomatopée en termes de sens. En outre, nous avons observé que certaines onomatopées ont été perçues par le public comme étant des représentations de stéréotypes négatifs liés à l'éducation des enfants, ce qui a donné lieu à une sorte de « contrôle pédagogique » de leur part.

Mots-clés : traduction, onomatopée, albums pour tout-petits, illustration, littérature de jeunesse

\footnotetext{
Abstract

Contemporary Conceptualisations of Translation Activity Proposed by Representatives of French Translation Enterprises

In this article, we examine to what extent the traditional conceptualisation of translation activity, i.e. the interlingual translation, has been enriched recently by elements of intralingual and intersemiotic translation. In the exploratory stage of the study, carried out in the late 2015, interviews with representatives of five French translation enterprises were analysed.
} 
The study reveals elements that demand an enlargement of the concept of translation: new transcreation service, audiovisual translation assignments and need for reformulating poorly written texts before translation.

Key words : translation, conceptualisation of translation activity, translation services, translation enterprise 